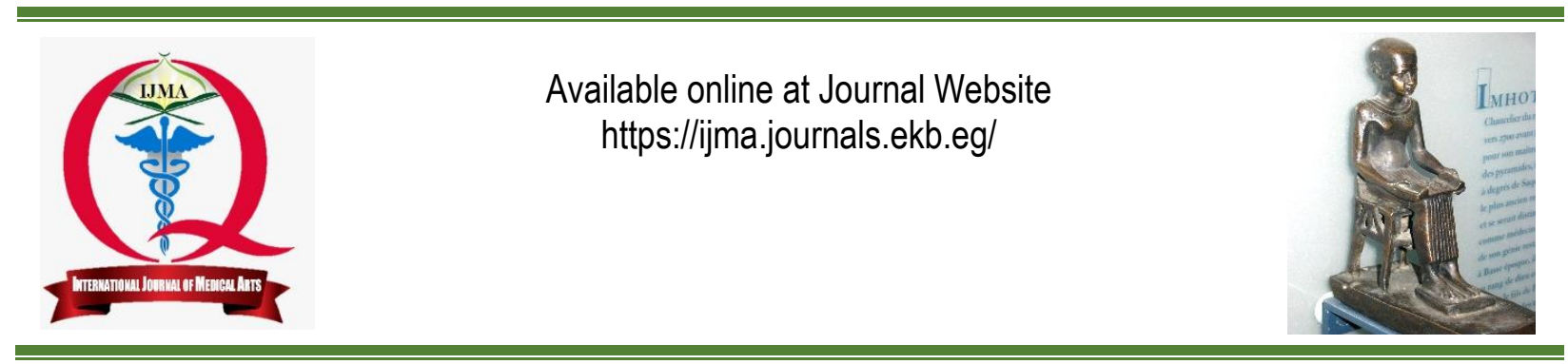

Original article

\title{
Evaluation of Vitamin D3 level in Sino-Nasal Polypi
}

\author{
Ali Abd-Allah Abdel-Rahmana; Mahmoud El-Said El-Sobkya; Amr Ahmed Rezkb; Mohamed Salah Emaraa \\ Department of Otorhinolaryngology, Dameitta Faculty of Medicine, Al-Azhar University, Egypt [a] \\ Department of Otorhinolaryngology, Faculty of Medicine, Al-Azhar University, Egypt [b] \\ Corresponding author: Mohamed Salah Emara \\ Email: dr.abusalah14@gmail.com
}

Received at: September 20, 2019; Revised At: October 11, 2019.

Accepted at: October 11, 2019. ; Available online at: October 11, 2019.

DOI: 10.21608/ijma.2019.17143.1030

\section{ABSTRACT}

Background: Vitamin D is a group of fat-soluble corticosteroid responsible for increasing intestinal absorption of calcium, magnesium, and phosphate, and multiple other biological effects. In humans, the most important compounds in this group are vitamin D3 [also known as cholecalciferol] and vitamin D2 [ergocalciferol].

Aim of the work: The aim of this thesis is to assess Vitamin D3 [VD3] level in patients with sino-nasal polypi in comparison with normal individuals.

Patients and Methods: This prospective comparative [case-control] study conducted by the Department of ENT, Al-Azhar University Hospitals in the period from March 2019 to September 2019 and included 40 individuals who were selected by inclusion and exclusion criteria, both genders, all patients had been counseled about the nature of the study and informed written consent has been obtained.

Results: Serum level of VD3 in patients with chronic rhinosinusitis with sinonasal polyps [CRSwNP] and allergic fungal rhinosinusitis [AFRS] was significantly lower than that of control subjects. Vitamin D levels ranged from 8.74 to $39 \mathrm{ng} / \mathrm{ml}$, and there was statistically significant decrease of serum vitamin $D$ levels in patient group when compared to control group [ $16.20 \pm 4.29$ vs $30.56 \pm 4.83$ respectively]. In addition, there was significant increase of patients with deficient and insufficient vitamin $D$ levels in patient group when compared to control group.

Conclusion: VD3 may constitute an inexpensive prophylactic and cost effective option in the therapeutic armamentarium in reducing inflammation either by itself or as a synergistic agent to traditional agents in patients suffering from sino-nasal polypi.

Keywords: Vitamin D3, Sino-nasal Polypi, Chronic rhinosinusitis; Allergic fungal rhinosinusitis.

This is an open access article under the Creative Commons license [CC BY] [https://creativecommons.org/licenses/by/2.0/]

Please cite this article as: Abdel-Rahman AA, El-Sobky ME, Rezk AA, Emara MS. Evaluation of vitamin D3 level in sino-nasal polypi. IJMA 2019; 1[2]; 132-137. 


\section{INTRODUCTION}

Vitamin D3 [VD3] is a potent immunemodulatory steroid hormone involved in the regulation of a variety of cell types that play a role in the local immune response [1]. Vitamin D3 [VD3] status is traditionally linked with rickets in children and osteomalacia in adults. However, levels of [VD3] have been recently found to be responsible for a number of immunological, anti-inflammatory and anti-infectious roles [2]. Some studies highlighted the potential impact of impaired VD3 status with respect to so-called "nonclassical" effects of VD3. These include cardiovascular actions, an antineoplastic agent, in prevention and/or treatment of infections and in association with the immune system [3].

Allergic fungal rhino-sinusitis [AFRS] is a distinct subtype of chronic rhino-sinusitis [CRS] that arises as a result of a localized allergic reaction to noninvasive fungal growth in areas of compromised mucus drainage [4]. Some forms of allergic fungal rhinosinusitis [AFRS], occurs more commonly in patients who are more susceptible to VD3 deficiency providing more evidence to support a role of VD3 as a key player in the immunopathology of chronic rhinosinusitis [CRS] with nasal polyps [CRSwNP] and AFRS [5]. It is important to consider that Vitamin $D$ insufficiency is a prevalent health problem all over the world, and this may partly explain the rising incidence of AFRS and other sinus problems [6].

\section{AIM OF THE WORK}

The aim of this thesis is to assess Vitamin D3 [VD3] level in patients with sino-nasal polypi in comparison with normal individuals.

\section{PATIENT AND METHOD}

This prospective comparative study conducted by the Department of ear, nose and throat [ENT] diseases, Al-Azhar University, in the period from March 2019 to September 2019 and included 40 of both genders, who had been counseled for the nature of the study and informed written consent.

Eligible patients were divided into three groups: Group A: included ten patients with allergic fungal rhinosinusitis according to the following criteria [Nasal polyposis; eosinophilic mucin; computed tomography [CT] scan findings consistent with chronic fungal rhinosinusitis; histological presence of fungal hyphae or positive fungal culture]. Group B: included ten patients with chronic rhinosinusitis with sinonasal polyposis diagnosed by CT and endoscopic examination plus postoperative histopathological examination of the polyps. Group $C$ is the control group consisting of twenty healthy volunteers with no history of sinusitis and no endoscopic evidence of inflammatory sinus disease at the time of the study. Group A and group $B$ were subjected to functional endoscopic sinus surgery [FESS] to obtain an excisional biopsy for histopathological examination.

Exclusion criteria: Patients who had received any form of immunotherapy, or vitamin supplements within 30 days before the surgery were excluded from the study. The following data has been collected: Detailed history taking; ENT examination plus through endonasal sinoscopic examination; CT paranasal sinuses and plain chest $X$-ray [except for controls]; $\mathrm{CBC}$ including blood eosinophils, renal function tests, liver function tests, and blood sugar.

Finally, all tissue samples obtained during surgery collected and sent for histopathological examinations and mycological work up in form of: Direct film examination for detection of the septate form of fungal infection; Silver stain [Gomori methenamine-silver]; Cultures were done on nutrient agar and Sabouraud agar.

Measurement of serum level of vitamin $D$ has been done by enzyme-linked immunosorbent assay [ELISA] using an ELISA kit for Human Vit D Immunoassay. All measurements were conducted in the same laboratory, by the same researcher, with the same kit. The blood samples were centrifuged for 15 minutes at $1250 \mathrm{~g}$. Subsequently, sera were aliquotted and frozen at $-70^{\circ} \mathrm{C}$ until the day of assay. Serum levels of $25[\mathrm{OH}]$ vitamin $D$ [VD3] were measured by using a VD3 enzyme immune assay kit [Catalog No: VD220B; Calbiotech]. Based on the principle of solid phase competitive enzyme-linked immunoassay. The test results are reported in nanogram per milliliter [ng/ml]. In addition, erum calcium [Ca] and phosphate [Po4] parameters has been measured.

Statistical methods: Data were analyzed using IBM SPSS Statistics version 21 [IBM Corp., Armonk, NY] and MedCalc version 12.5 [MedCalc 
Software bvba, ostend, Belgium]. The D'AgostinoPearson test was used to examine the normality of numerical data distribution. Normally distributed numerical variables are presented as mean \pm standard deviation [SD]; intergroup differences were compared using One- way analysis of variance [ANOVA] with application of the Scheffe test for Post Hoc comparisons. The Kruskal-Wallis test was used to compare non-normally distributed numerical variables and the Conover test was used for post hoc comparisons. The Pearson chi-square test, or Fisher's exact test when appropriate, was used to compare nominal data. Multinomial logistic regression was used to examine the relation between VD3, Calcium level, phosphate level, and the type of chronic sinusitis, adjusting for the effect of possible confounders. All tests were 2-tailed. Values of $p<0.05$ were considered statistically significant.

\section{RESULTS}

The study included 40 participants. They were categorized into 2 groups. 20 patients in the patients group; 11 of them [55.0\%] were males and 9 of them [45.0\%] were females. In addition, it include 20 healthy subjects as a control group, 13 [65.0\%] were males and 7 [35.0\%] were females; and there was no significant difference between patients and control groups. Furthermore, the age of participants ranged from 23 to 60.0 years; and there was no significant difference between the patients and control groups $[39.20 \pm 9.26$ vs $35.05 \pm 9.75$ years respectively].

In the present work, vitamin $D$ levels ranged from 8.74 to $39 \mathrm{ng} / \mathrm{ml}$, and there was statistically significant decrease of serum vitamin $D$ levels in patients when compared to control group [16.20 \pm 4.29 vs $30.56 \pm 4.83$ respectively]. In addition, there was significant increase of patients with deficient and insufficient vitamin $D$ levels in patients when compared to control group. In patient group, $80.0 \%$ had deficient, while $20.0 \%$ had insufficient vitamin $D$ levels. In control group, 5.0\% had deficient, $45.0 \%$ had insufficient and $50.0 \%$ had sufficient vitamin D levels [Table 1].

When comparing CRSwNP and AFRS, there was no significant difference regarding nasal obstruction and epistaxis, while anosmia was significantly increase in AFRS when compared to CRSwNP [ $100.0 \%$ vs $60.0 \%$ respectively] [table 2].

Results of the CBC showed non-significant difference between CRSwNP and AFRS groups, except significant increase of eosinophil count in CRSwNP when compared to AFRS groups [297.00 \pm 88.05 vs $151.40 \pm 48.56$ respectively] [table 3]. Serum urea was significantly reduced in CRSwNP when compared to AFRS groups $[20.90 \pm 0.18$ vs $25.50 \pm 4.81$ respectively]. In addition, AST significantly lower in CRSwNP when compared to AFRS groups [20.0 \pm 5.91 vs $24.80 \pm 2.75$ respectively]. However, the values in both groups were in normal range. Otherwise, there was no significant difference between both groups regarding other liver or kidney function tests [table 4]. Patients with CRSwNP had significantly higher calcium and lower phosphate when compared to AFRS $[8.44 \pm 0.61, \quad 2.07 \pm 0.50$ vs $7.75 \pm 0.11$, $2.46 \pm 0.16$ respectively] [table 5]. In addition, serum levels of vitamin $D$ showed no significant difference between CRSwNP and AFRS groups. However, when categorizing levels of vitamin $D$, patients with CRSwNP had significant increase of insufficient vitamin D when compared to AFRS group [ $40.0 \%$ vs $0.0 \%$ ] [Table 6]. Serum vitamin D correlates significantly in an inverse manner with eosinophil count, while the correlation with calcium and phosphate levels was proportional [table 7].

Table [1]: Vitamin D levels among studied groups

\begin{tabular}{|c|l|c|c|c|c|}
\hline \multicolumn{2}{|c|}{ Variable } & Patients group & Control group & Test & P value \\
\hline \multirow{2}{*}{ Vitamin D [ng/ml] } & $\begin{array}{c}16.20 \pm 4.29 ; \\
8.74-27.0\end{array}$ & $\begin{array}{c}30.56 \pm 4.83 ; \\
19.91-39.0\end{array}$ & \multirow{2}{*}{9.93} & \multirow{2}{*}{$0.001^{*}$} \\
\hline \multirow{3}{*}{ Categories } & Deficient & $16[80.0 \%]$ & $1[5.0 \%]$ & \multirow{2}{*}{25.15} & \multirow{2}{*}{$0.001^{*}$} \\
\cline { 2 - 4 } & Insufficient & $4[20.0 \%]$ & $9[45.0 \%]$ & \\
\cline { 2 - 4 } & Sufficient & $0[0.0 \%]$ & $10[50.0 \%]$ & & \\
\hline
\end{tabular}


Abdel-Rahman et al.

Table [2]: Comparison between CRSwNP and AFRS regarding clinical presentation among studied groups

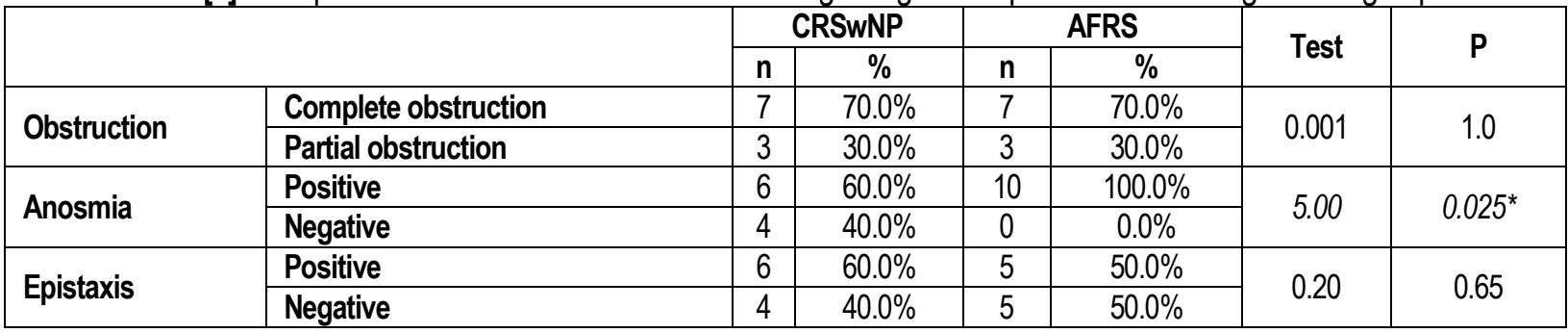

Table [3]: Comparison between CRSwNP and AFRS regarding results of complete blood count among studied groups

\begin{tabular}{|l|c|c|c|c|c|c|}
\hline \multirow{2}{*}{ Variable } & \multicolumn{2}{c|}{ CRSwNP } & \multicolumn{2}{c|}{ AFRS } & \multirow{2}{*}{ Test } & \multirow{2}{*}{ P value } \\
\cline { 2 - 6 } & Mean & SD & Mean & SD & & 0.41 \\
\hline Hemoglobin [g/dl] & 11.56 & 0.58 & 11.30 & 0.78 & 0.83 & 0.17 \\
\hline HCT\% & 39.42 & 2.20 & 37.80 & 2.85 & 1.42 & 0.42 \\
\hline RBCs x 106 & 3.87 & 0.22 & 3.79 & 0.25 & 0.81 & 0.11 \\
\hline WBCs x 103 & 8.13 & 2.08 & 6.70 & 1.69 & 1.68 & 0.94 \\
\hline Platelets x 103 & 227.00 & 22.38 & 228.10 & 43.57 & 0.07 & $0.001^{*}$ \\
\hline Eosinophil count & 297.00 & 88.05 & 151.40 & 48.56 & 4.57 & 0 \\
\hline
\end{tabular}

Table [4]: Liver and kidney function tests among studied groups

\begin{tabular}{|l|c|c|c|c|c|c|}
\hline \multicolumn{1}{|c|}{ Variable } & \multicolumn{2}{c|}{ CRSwNP } & \multicolumn{2}{c|}{ AFRS } & Test & P value \\
\hline Urea [mg/dl] & 20.90 & 4.20 & 25.50 & 4.81 & 2.27 & $0.035^{*}$ \\
\hline Creatinine [mg/dl] & 0.77 & 0.18 & 0.92 & 0.15 & 2.06 & 0.06 \\
\hline ALT & 27.40 & 8.33 & 24.50 & 3.31 & 1.02 & 0.32 \\
\hline AST & 20.00 & 5.91 & 24.80 & 2.78 & 2.32 & $0.032^{*}$ \\
\hline Total bilirubin [mg/dl] & 0.76 & 0.22 & 0.75 & 0.30 & 0.08 & 0.93 \\
\hline Albumin [g/dl] & 4.15 & 0.28 & 3.99 & 0.40 & 1.02 & 0.31 \\
\hline
\end{tabular}

Table [5]: Serum calcium and phosphate levels among studied groups

\begin{tabular}{|c|c|c|c|c|c|c|}
\hline \multirow{2}{*}{ Variable } & \multicolumn{2}{|c|}{ CRSwNP } & \multicolumn{2}{|c|}{ AFRS } & \multirow{2}{*}{ Test } & \multirow{2}{*}{ P value } \\
\cline { 2 - 7 } & Mean & SD & Mean & SD & & $0.002^{*}$ \\
\hline Calcium [mg/dl] & 8.44 & 0.61 & 7.75 & 0.11 & 3.54 & $0.014^{*}$ \\
\hline Phosphate & 2.07 & 0.50 & 2.46 & 0.16 & 1.39 & ${ }^{*}$ \\
\hline
\end{tabular}

Table [6]: Vitamin D levels among studied groups

\begin{tabular}{|c|l|c|c|c|c|c|c|}
\hline \multicolumn{2}{|c|}{ Variable } & \multicolumn{2}{c|}{ CRSwNP } & \multicolumn{2}{c|}{ AFRS } & Test & P value \\
\hline \multicolumn{2}{|c|}{ Vitamin D } & 17.61 & 5.14 & 14.78 & 2.84 & 1.52 & 0.14 \\
\hline \multirow{2}{*}{ Categories } & Deficient & 6 & $60.0 \%$ & 10 & $100.0 \%$ & \multirow{2}{*}{5.0} & \multirow{2}{*}{$0.025^{\star}$} \\
\cline { 2 - 8 } & Insufficient & 4 & $40.0 \%$ & 0 & $0.0 \%$ & & \\
\hline
\end{tabular}

Table [7]: Correlation between vitamin $\mathrm{D}$ and descriptive variables among studied groups

\begin{tabular}{|l|c|c|}
\hline \multirow{2}{*}{ Age } & \multicolumn{2}{|c|}{ Vitamin D } \\
\cline { 2 - 3 } & \multicolumn{2}{|c|}{$\boldsymbol{P}$} \\
\hline Hemoglobin & -.185 & .252 \\
\hline Hct & -.052 & .749 \\
\hline RBCS & .017 & .915 \\
\hline WBCs & -.070 & .666 \\
\hline Platelets & -.297 & .062 \\
\hline EosinOphils & .029 & .857 \\
\hline Urea & $-.388^{*}$ & .013 \\
\hline Creatinine & .097 & .553 \\
\hline ALT & -.230 & .153 \\
\hline AST & .014 & .931 \\
\hline Total bilirubin & -.277 & .083 \\
\hline Albumin & -.287 & .072 \\
\hline Calcium & .063 & .700 \\
\hline Phosphate & $.858^{* *}$ & .000 \\
\hline Signifant & $.847^{* *}$ & .000 \\
\hline
\end{tabular}

*: significant at $p$ level $<0.01$; **: significant at $p$ level $<0.001$ 


\section{DISCUSSION}

This study showed that serum level Of VD3 in patient with sinonasal polyposis either CRSwNP or fungal is significantly lower than that of the control subjects [ $p<0.001]$. The mean vitamin $D$ levels in patients when compared to control group [16.20 \pm 4.29 vs $30.56 \pm 4.83 \mathrm{ng} / \mathrm{ml}$ respectively]. In addition, there was significant increase of patients with deficient and insufficient vitamin $D$ levels in patients when compared to control group. In patients group, $80.0 \%$ had deficient, while $20.0 \%$ had insufficient vitamin D levels. In control group, $5.0 \%$ had deficient, $45.0 \%$ had insufficient and $50.0 \%$ had sufficient vitamin D levels.

In accordance with Mulligan et al., they stated that both CRSwNP and AFRS patients were identified as being VD3-insufficient compared to control and CRSsNP. [22.1 $\pm 3.2 \mathrm{ng} / \mathrm{ml}$ and $38.4 \pm 3.2$ $\mathrm{ng} / \mathrm{ml}$, respectively] [5]. Also McCarty et al. reported that low VD3 levels were documented in patients with chronic CRSwNP, and VD3 was shown to inhibit proliferation of nasal-polyp fibroblasts that may confirm its anti-inflammatory effect ${ }^{[7]}$. Mostafa et al. ${ }^{[8]}$ study also showed that serum level of VD3 in patient with CRSwNP and AFRS is significantly lower $[p<0.001]$ than that of patients with CRSsNP and control subjects.

On the other hand, in their studies, Apuhan et al. [9] could not prove any association between serum VD3 level and CRSwNP. Another study showed the circulating levels of 25-OH-VD3 were equivalent between patients with CRSwNP and control [10].

In our study, $70 \%$ of the patient group have a history of allergy with significant increase of positive history of chronic disease in patients group when compared to control group. This result is in agreement with a study done by Wjst and Hypponen [11]; they found that the prevalence of allergic rhinitis increased across quartile groups of low VD3 serum levels.

Our study showed that VD3 levels were not influenced by gender or age among the groups studied. However, in some ways it is not surprising that there are some arguments concerning the systemic 25-OH-VD3 status in patients with CRSwNP from different races. Pinto et al. [12] showed significantly lower serum VD3 levels in African Americans with CRS compared with race and sex-matched controls. But in our study no multiple races were involved as it was not available and the number of the cases were limited.

In our study there was a significant decrease of serum calcium in patients when compared to control group [ $8.09 \pm 0.55$ vs $9.71 \pm 0.58 \mathrm{mg} / \mathrm{dl}$ respectively]. On the other side, serum phosphate showed significant decrease in patients when compared to control group $[2.26 \pm 0.41$ vs $2.93 \pm 0.43$ respectively]. And this could be attributed to absence the effect of vitamin $D$ in absorption of calcium and phosphate from GIT and increase phosphate excretion through the kidney mediated by parathormone hormone released in response to calcium deficiency. This result was correspondent with a study done by Mostafa et al. ${ }^{[8]}$, as there was also a significant decrease in the serum $\mathrm{PO} 4$ level in patient groups compared with control groups This can be explained by the following: vitamin $D$ deficiency stimulates the parathyroid glands, leading to secondary hyperparathyroidism, which maintains serum calcium in the normal range at the expense of mobilizing calcium from the bone and increases urinary phosphate loss, resulting in a decrease in serum phosphate level.

On the contrary, no relationships were detected between levels of serum ionized calcium, phosphate, and serum vitamin D metabolites in the study conducted by Brot et al. [13].

In our study there was significant increase of WBCs and eosinophil count in patient when compared to control group $\left[7.41 \pm 1.99 \times 10^{3}\right.$, $224.20 \pm 101.85$ vs $6.07 \pm 1.56 \times 10^{3}$ and $104.25 \pm 61.01$ respectively]. And this may be due to presence of allergy or fungal infection in our study groups. However, there is no evidence of relationship between eosinophilia and vit $D$ deficiency. And this coordinated with Mulligan et al. as they were unable to identify a correlation between peripheral eosinophilia and VD3 levels [5].

In another study, Faruk et al. [14] suggests that, when using systemic VD3 in high therapeutic dose, it reduces the size of nasal polyp and facilitates the restoration of nearly normal sinonasal mucosa. Hence, normalization of VD3 level may result in clinical improvement due to restoration of its antiinflammatory role in the body.

The above studies along with our investigation highlight the fact that there are still many controversies regarding vitamin $D$ status in CRS. 
In conclusion, VD3 may constitute an inexpensive prophylactic and cost effective option in the therapeutic armamentarium in reducing inflammation either by itself or as a synergistic agent to traditional agents in patients suffering from sino-nasal polypi. However, because there is no consensus on the serum vitamin $D$ levels to consider appropriate for global health, the doses to use for its supplementation, or the cutoffs for its deficiency, we are in need of large, controlled, prospective studies on VD3 supplementation to clarify whether it has a role in the pathogenesis, prevention of and treatment for AFRS, CRSwNP, and allergic conditions.

\section{Conflict of Interest}

Authors declare that, there was no conflicts of interest.

\section{REFERENCES}

1. Brehm JM, Celedon JC, Soto-Quiros ME,Avila L, Hunninghake GM, Forno $E$, et al. Serum vitamin D levels and markers of severity of childhood asthma in Costa Rica. Am J Respir Crit Care Med. 2009; 179:765771. [DOI:10.1164/rccm.200808-13610C].

2. Holick MF. Vitamin D status: measurement, interpretation, and clinical application. Ann Epidemiol. 2009; 19:73-78.[DOI:10.1016/j.annepidem. 2007. 12.001].

3. Zittermann A. Vitamin D and disease prevention with special reference to cardiovascular disease. Prog Biophys Mol Biol Cancer 2013; 5:10721085.[DOI:10.1016/j.pbiomolbio.2006.02.001].

4. Luong A, Marple BF. Allergic fungal rhinosinusitis. Curr Allergy Asthma Rep. 2004; 4[6]: 46570.[PMID:15462713].

5. Mulligan JK, Bleier BS, O'Connell B, Mulligan RM, Wagner C, Schlosser RJ. Vitamin D3 correlates inversely with systemic dendritic cell numbers and bone erosion in chronic rhinosinusitis with nasal polyps and allergic fungal rhinosinusitis. Clin Exp Immunol. 2011; 164:312-320.[DOI:10.1111/j.1365-2249.2011.04325.x].
6. Ginde AA, Mansbach JM, Camargo CA. Association between serum 25 hydroxyvitamin $D$ level and upper respiratory tract infection in the Third National Health and Nutrition Examination Survey. Arch Intern Med. 2009; 169:384-390.[10.1001/archinternmed. 2008. 560].

7. McCarty DE, Chesson AL, Jain SK, Marino AA. The link between vitamin $D$ metabolism and sleep medicine. Sleep Med Rev. 2014; 18:311-319. [DOI:10.1016/j.smrv.2013.07.001]

8. Mostafa BE, Taha MS, Abd-Ei Hamid T, Omran A, Lotfi N. Evaluation of vitamin D levels in allergic fungal sinusitis, chronic rhinosinusitis, and chronic rhinosinusitis with polyposis. Int Forum Allergy Rhinol. 2015; 6[2]:185-90.[DOI:10.1002/alr.21585].

9. Apuhan T, Bugdaycı G, Alcelik A, Aktas G. Serum levels of vitamin $D$ among patients with nasal polyps in Bolu, Turkey. J Aller Ther. 2011; 5:001. [10.4172/ 21556121.s5-001].

10. Erdag O, Turan M, Ucler R, Berkoz M, Garca MF, Bozan N, Kıroglu AF, Cankaya H. Is nasal polyposis related to levels of serum vitamin $D$ and vitamin $D$ receptor gene expression? Med Sci Monit. 2016; 22:4636-43.[DOI:10.12659/msm.898410].

11. Wjst M, Hypponen E. Vitamin D serum levels and allergic rhinitis. Allergy 2007; 62:1085-1086. [DOI:10.1111/j.1398-9995.2007.01437.x]

12. Pinto JM, Schneider J, Perez R, De Tineo M, Baroody FM, Naclerio RM. Serum 25-hydroxyvitamin D levels are lower in urban African American subjects with chronic rhino-sinusitis. J Allergy Clin Immunol. 2008; 22:415-7.[10.1016/j.jaci.2008.05.038].

13. Brot C, Jørgensen N, Madsen OR, Jensen LB, Sørensen $\mathrm{OH}$. Relationships between bone mineral density, serum vitamin D metabolites and calcium: phosphorus intake in healthy perimenopausal women. J Intern Med. 1999; 10:509-516.[DOI:10.1046/j.13652796.1999.00474.x].

14. Faruk EM, Yousef MM, Mohamed T. Does vitamin D have protective effect on human nasal polyposis: histological and immunohistochemical study. J Histol Histopathol. 2014; 1:2.[10.7243/2055-091X-1-2]. 